\title{
The relationship between Dispositional Optimism, Pain Experience and Pain anticipation
}

\author{
M.H.M. Klomp \\ Maastricht University \\ mhm.klomp@student.maastrichtuniversity.nl
}

\begin{abstract}
Background: Dispositional optimism is an individual difference variable that reflects the extent to which people hold generalized favorable expectancies for their future. It has been suggested to be a protective factor for pain. This study investigates the relationship between dispositional optimism and pain experience, and what role pain anticipation plays in this relationship. A positive impact of dispositional optimism on pain experience is expected. Furthermore, it is expected that pain anticipation has a negative association with both dispositional optimism and pain experience, but also may be a mediator in the relationship between dispositional optimism and pain experience. Methods: The study has a cross-sectional design and was performed on 36 bachelor students of Maastricht University. The study population has an age ranged from 18 to 24 years old (mean=20,75 years; $S D=1,84$ ). Experimental pain was induced by means of the Medoc Pathway Advanced Thermal Stimulator (ATS; MEDOC). Pain experience was separated in pain threshold and pain tolerance measurements. Dispositional optimism was measured with the Life Orientation Test-Revised (LOT-R). Pain anticipation was separated in three items; expected pain, expected unpleasantness, and expected fear, which were measured on a visual analogue scale before the start of the pain stimulus. Results: For pain anticipation and pain experience only expected pain shows a significant correlation with pain experience's measurement pain tolerance. There is no significant link between dispositional optimism and pain experience; therefore, pain anticipation is not tested as a mediator. In the correlation between pain anticipation and dispositional optimism, the link between expected pain and the total LOT-R score is significant. Furthermore, the links between expected pain, expected unpleasantness and expected fear and the negative subscale of the LOT-R score are significant. Conclusion: This study did not confirm the suggested relationship between dispositional optimism and pain experience, although it does confirm a significant correlation between pain anticipation and dispositional optimism.
\end{abstract}




\section{Keywords}

Dispositional optimism, Pain experience, Pain threshold, Pain tolerance, Pain anticipation.

\section{Introduction}

Pain can be defined as an unpleasant sensory and emotional experience, which is associated with current or potential tissue damage, or described in terms of such damage (1). It is a defending mechanism to protect the body from tissue damage. The stimuli of pain are transferred to the thalamus, which projects the signal to several regions like other basal brain parts and the somatosensory cortex (2). Even though the biological pathways of pain are the same in each person, pain experience is not equal for everyone.

According to the biopsychosocial perspective, a multidimensional perspective on pain, human pain experience is the result of the interaction between psychological, social, physiological and behavioural factors (4). The experience of pain is thus highly subjective and can vary substantially from one individual to the next (3). Due to the biopsychosocial perspective the evidence that psychological factors play an important role in the pain experience started to grow and the psychology of pain proved to be a fruitful area of research (5). Researchers started to investigate the role of positive personality traits and emotions as a determinant in the experience of pain (6). One of these positive personality traits that has a positive effect on the experience of pain proved to be dispositional optimism.

Dispositional optimism is an individual difference variable that reflects the extent to which people hold generalized favorable expectancies for their future (8). This means that optimists have a positive mindset and expect good things to happen to them. When confronted with pain, optimists show better adjustment and less pain-sensitivity (7, 9, 10). This personality trait is linked with lower reports of clinical pain and less severe pain elicited in laboratory settings.

Also in the last couple of years research proposes that, besides optimism, pain experience, is also influenced by expectations. When the sensory information is in the form of a pain stimulus, expectations will be interpreted as pain anticipation. Anticipation of pain is a complex state that influences the immediate unpleasantness of pain (13). According to Angst, Tingle, Phillips and Carvallo (2009), there is a strong correlation between pain anticipation and the intensity of pain experience. Positive anticipation can powerfully reduce the pain experience, whereas negative anticipation may result in aggravation of pain feelings (14-16). 
Besides its effect on pain experience, it may be proposed that pain anticipation also is a mediating factor in the association between dispositional optimism and the experience of pain (5). Research suggests that dispositional optimism interacts with situational factors in determining pain sensitivity. Because of the positive expectations that hold optimistic for their future, it is possible that these expectations also extend to painspecific expectations, thereby diminishing pain-sensitivity and avoidance behavior (5).

Even though it seems logical that optimistic people have more positive pain-specific expectations and pain anticipation may be a mediating factor, not much research has been done about the separate relationship between pain anticipation and dispositional optimism. Previous research only focused on negative personality traits.

To build further on previous research, the current study will examine how optimism levels are associated to pain experience by inducing heat pain in pain free healthy participants. In addition it will be examined if expectancies that people hold about an upcoming pain experience are contributing to the pain experience as well, thereby mediating the relationship between optimism and pain experience. In addition, the association between optimism levels and the expectancies about pain, also called pain anticipation levels will be examined.

By indicating the possible correlations between optimism, pain experience and pain anticipation, the results may be used for designing and constructing pain treatment therapies or programs for painful interventions. To confirm and understand these associations these interactions a research question was formulated for this study; is dispositional optimism related to experimental pain experience and is this relation influenced by pain anticipation in healthy bachelor students of Maastricht University? On the basis of previous research, the hypotheses for this study were drawn:

- The level of dispositional optimism is positive correlated with pain experience, namely increased pain threshold and pain tolerance.

- The pain anticipation scores according to the items expected pain, expected unpleasantness and expected fear are negative correlated with pain experience, namely increased pain threshold and pain tolerance.

- The pain anticipation scores according to the items expected pain, expected unpleasantness and expected fear are mediating factors in the relation between dispositional optimism and pain experience. 
- Pain anticipation, namely scores on expected pain, expected unpleasantness and expected fear is negative correlated with the level of dispositional optimism.

\section{Materials and methods}

\section{Participants}

Participants were recruited from the student population of Maastricht University. Inclusion criteria included: Dutch bachelor students with an age of 18 years and older. Exclusion criteria included: pregnancy, having a cardiovascular disease or having a psychological disorder. All participants gave written informed consent to participate in this study. All procedures and protocols were approved by the Ethical Committee of the faculty of Psychology and Neuroscience of Maastricht University. Thirty-six participants, nine males and 27 females, ranged in age from 18 to 27 years ( mean $=20,75$ years; SD $=1,84$ ) volunteered to participate in this study in exchange for course credits or a small financial compensation.

\section{Measures and materials}

\section{Apparatus}

Experimental pain was induced by means of the Medoc Pathway Advanced Thermal Stimulator (ATS; MEDOC). This is a precise, computer-controlled device that is capable of generating and documenting response to highly repeatable thermal stimuli (17). It delivers heat stimuli to the skin via a thermode, which contains a metal plate $(3 \times 3 \mathrm{~cm})$. The thermode with the metal plate was attached two to three centimetres from the wrist to the inner side of the forearm of the non-dominant hand. The heat stimuli were generated and transmitted from the central unit and controlled by software that was installed on a laptop (18). The basal thermode temperature was set to $32^{\circ} \mathrm{C}$ and could rise to the maximum of $51^{\circ} \mathrm{C}(18)$. Also attached to the central unit was a hand-held device that gave the participants the opportunity to stop the heat stimulus at any time.

\section{Pain variables; pain threshold and pain tolerance}

The pain threshold indicates the level of temperature at which the participant first feels pain and can be defined as; 'the point between just about to be painful and just became painful'\{Angst, $2009 \# 15\}$ (20). When the heat stimuli started the metal plate started to rise in temperature, $1^{\circ} \mathrm{C} / \mathrm{sec}$, until the point that the participant experienced first feeling of pain, the point between just about to be painful and just became painful, and stopped the stimulus with the hand-held device. 
The pain tolerance indicates the level of temperature at which the participant indicates that he or she can no longer cope with the pain and is not prepared for more intense pain. When the heat stimuli was started the metal plate started to rise in temperature, $1^{\circ} \mathrm{C} /$ $\mathrm{sec}$, until the point that the participant could no longer cope with the pain and stopped the stimulus with the hand-held device. Immediately after the participant stopped a stimulus the temperature was registered and dropped with $8^{\circ} \mathrm{C} / \mathrm{sec}$ to the basal thermode temperature. The pain threshold measure and the pain tolerance measure were both assessed three times.

\section{Pain ratings; anticipation and pain experience}

The pain anticipation measurement consisted of four questions that were answered on a visual analogue scale (VAS). The aspects that were questioned in the pain anticipation measurement were about the expected degree of pain of the stimulus, unpleasantness of the stimulus, fear for the stimulus, and fear for the pain of the stimulus. Because the last two questions can be highly correlated they form together the mean item expected fear. The questions about expected pain and expected unpleasantness each formed a separate item in the scoring of the pain anticipation construct.

Pain experience was measured four times during the pain inductions. The first measurement consisted of four questions that were about the experience of four different aspects, namely the degree of pain of the stimulus, the degree of unpleasantness of the stimulus, the degree of fear for the stimulus, and the degree of fear for the pain of the stimulus. The second, third and fourth measurement consisted of two questions that were about the experience of the aspects; the degree of pain of the stimulus and the degree of fear for the pain of the stimulus. All pain experience questions were also answered on a visual analogue scale (VAS).

\section{Questionnaires; personality constructs}

The LOT-R questionnaire was used to measure dispositional optimism. Scheier and Carver (1985) developed the Life Orientation Test-Revised (19). This self-report evaluates generalized expectations of positive and negative outcomes (21). It contains four filler items and six items that are used to derive the total optimism score. The six items consisted of three items that are negatively phrased and three items that are positively phrased. Participants rate each item with the extent of their agreement from o (strongly disagree) to 4 (strongly agree) (19). The LOT-R is stable over time, had a normal to high internal consistency and showed unique predictive value over self-mastery, self-esteem 
and trait anxiety (19). The Cronbach's alpha for the LOT-R total score for this study was 0,82. The Cronbach's alpha for the positive subscale was 0,64 and 0,73 for the negative subscale.

\section{Procedure}

The participant took place in a private room with a quiet and calm area. The researcher gave the participant a small introduction and the overall instructions of the experimental procedure. Then the participant received an information letter and an informed consent. Instructions about the threshold pain inductions were given to the participant. If the participant did not have any questions, the thermode was attached with Velcro to the wrist. The participant completed four pain anticipation questions before the start of the stimuli. The heat stimulus was started by the researcher and stopped by the participant. The procedure for threshold pain induction was repeated three times. Between the stimuli there was a break of twenty seconds. The researcher warned the participant each time a stimulus started. After the three stimuli, the participant completed four questions about pain experience. The instructions for the pain tolerance inductions were given. If there were not any questions, the participant completed four questions about pain anticipation before the start of the stimuli. The procedure for tolerance was also repeated three times. Between each stimulus the participants completed two questions about pain experience in the time of twenty seconds. After the three stimuli, the participant completed another two questions about pain experience. The researcher removed the TSA thermode of the wrist of the participant and gave the instruction to complete the LOT-R questionnaire on the computer. Besides the LOT-R questionnaire the study involved eight other questionnaires; ISI, HSPS, ASI, PCS, MHLC, general anxiety illness and injury, BIS/BAS and FPQ, but these were disregarded in this paper.

\section{Data collection}

Data was collected via the use of the software from the TSA and the program EMIUM. The software from the TSA 2001 registered the temperature of the thermode immediately after the participant pushed the stop button on the hand-held device. The program EMIUM registered the answers of the pain anticipation questions, the pain experience questions and the nine questionnaires that were entered in an SPSS data file.

\section{Statistics}

Data were analyzed using SPSS version 21.0. First the characteristics of the group of thirtysix students were defined and the descriptives were calculated. For the analysis the scores of the LOT-R questionnaire, the mean pain threshold and tolerance and the items of pain 
anticipation were used. The data was checked on normality and outliers. Furthermore, Cronbach's alpha of the LOT-R test was calculated. The scores of the items 3, 7, and 9 of the LOT-R test were reversed before the total was calculated. The total score was calculated by the sum of item 1, 4, 10, and the reversed items 3,7 , and 9 . The LOT-R questionnaire has two subscales which were separated. The positive LOT-R subscale consisted of the items 1 , 4 , and $\mathrm{f}$. The negative LOT-R subscale consisted of the non-reversed items 3, 7, and 9. Data were analyzed using bivariate Pearson correlations between the dispositional optimism score and the pain threshold and pain tolerance, the items of pain anticipation and the pain threshold and the pain tolerance, and between the dispositional optimism scores and the items of pain anticipation scores on the VAS. Furthermore, pain anticipation was tested as a mediator of the possible relationship between dispositional optimism and pain experience via regressions with the method of Baron and Kenny (1986) (20). P values $<0,05$ were considered statistically significant.

\section{Results}

General descriptive

The sample had an age range of 18 to 27 years, with a mean of $20,75(S D=1,84)$ and was predominantly female ( $75 \%)$. The majority of the participants finished pre-university education and were second year students

\section{Pain experience and dispositional optimism}

Correlations between pain experience and dispositional optimism were calculated. Pain experience was separated into pain threshold and pain tolerance. Using a bivariate correlation analysis, the Pearson correlations between mean threshold and scores of LOT-R were calculated, but there were no significant correlations between both the score of dispositional optimism and pain threshold and the score of dispositional optimism and pain tolerance.

\section{Pain anticipation and pain experience}

Pain anticipation was divided in three items; expected pain, expected unpleasantness, and expected fear. Each item was correlated to pain experience, which was separated in pain threshold and pain tolerance. Using a bivariate correlation analysis, the Pearson correlations were calculated. All correlations between pain threshold and the three items of pain anticipation were very weak and not significant. In addition, for pain tolerance both expected unpleasantness and expected fear did also not correlate. Conversely, there was a significant and strong, negative correlation between the item expected pain and pain tolerance $(-0,43$, sig $(2$-tailed $)=0,01)$. 


\section{Pain anticipation as a mediator}

There is no direct significant link between the scores on the LOT-R questionnaire and the pain experience measurements. Therefore, testing the mediator test is not useful according to the method of Baron and Kenny (1986).

\section{Pain anticipation and dispositional optimism}

The last correlation that will be drawn is the correlation between the three separated items of pain anticipation and the score of dispositional optimism. In order to analyze more in detail the subscales of the LOT-R were also separately correlated to each pain anticipation item. Using a bivariate correlation analysis, the Pearson correlations were calculated. There was one significant, negative correlation between the total score on the LOT-R questionnaire and the item expected pain. This was a moderately strong correlation of - 0,37 . Other significant correlations were found between the negative subscale of LOT-R and all three pain anticipation items, expected pain $(0,43)$, expected unpleasantness $(0,37)$ and expected fear $(0,33)$.

\section{Discussion and conclusion}

The primary aims of the current study were 1 ) to examine whether there was a significant relationship between dispositional optimism and pain responses, 2) to examine whether there was a significant link between pain anticipation and pain experience, 3 ) to examine if pain anticipation had a mediator function in the relationship between dispositional optimism and pain experience, and 4) to examine whether there was a significant link between pain anticipation and dispositional optimism. Pain experience was divided in pain threshold and pain tolerance. Pain anticipation consisted of three separate items, namely expected pain, expected unpleasantness and expected fear.

The results do not show a significant (positive) relationship between dispositional optimism and pain threshold or pain tolerance (aim 1). An explanation for the non significant correlation between dispositional optimism and pain tolerance could be that this study has a small study population which was also not equal in numbers of man and woman.

Furthermore, the results about pain anticipation and pain experience do show a significant relationship between the expected pain and pain tolerance. The other correlations between the items of pain anticipation and pain threshold and pain tolerance do not show any significance (aim 2). An explanation for the fact that these items do not 
correlate with pain threshold and pain tolerance may be that the feeling of control that the participants had due to the fact that they could stop the stimulus at any time, influenced their expectations about the pain they would experience. Again the small sample size and the unequal numbers of men and women may also be an explanation for the fact that only the item expected pain is significantly linked to pain tolerance.

The results about pain anticipation as a mediator in the relationship between dispositional optimism and pain experience show that the mediation could not be tested, because that would not be useful if the link between dispositional optimism and pain experience is not significant (aim 3).

The last results about pain anticipation in relation to dispositional optimism showed a significant, negative correlation between expected pain and the total LOT-R score. In addition, the correlations between the three pain anticipation items, expected pain, expected unpleasantness, and expected fear and the negative subscale LOT-R score were significant (aim 4). A possible explanation for this could be that in the study of Hanssen (2014) pain was induced with a cold pressor instead of heat stimulation which was used in the current study.

According to the research question: 'is dispositional optimism related to experimental pain experience and is this relation influenced by pain anticipation in healthy bachelor students of Maastricht University?' could be stated that there was no relationship between dispositional optimism and pain experience, but there were significant links between pain anticipation and pain experience and between pain anticipation and dispositional optimism.

One area for further investigation would be the link between pain anticipation and dispositional optimism. Not much research has been done about this link yet, but the current study showed significant correlations between pain anticipation items and dispositional optimism scores. Therefore, it is interesting for future research.

\section{Role of the student}

The student was an undergraduate student working under the supervision of Linda Vancleef when the research in this report was performed. The topic was chosen by the student but the further research was designed by the supervisor. The collection of the participants, the processing of the results, the formation of the conclusion and the writing were done by the student. 


\section{References}

1. Merskey HDM. Respons to Editorial: New perspectives on the Definition of Pain. Pain. 1996.

2. Wilgen CP, Nijs J. Pijneducatie: een praktische handleiding voor (para)medici. Houten: Bohn Stafleu van Loghum; 2010.

3. Coghill RC, McHaffie JG, Yen YF. Neural correlates of interindividual differences in the subjective experience of pain. Proc Natl Acad Sci U S A. 2003;100(14):8538-42.

4. Ebert MH, Kerns RD. Behavioral and Psychopharmacologic Pain Management. Cambridge: Cambridge University Press; 2011.

5. Hanssen MMP. Optimism, the natural placebo: Cognitive, behavioural and motivational mechanisms of resilience towards pain (Doctoral dissertation). Maastricht: Maastricht University; 2014.

6. Goodin BR, Glover TL, Sotolongo A, King CD, Sibille KT, Herbert MS, et al. The association of greater dispositional optimism with less endogenous pain facilitation is indirectly transmitted through lower levels of pain catastrophizing. J Pain. 2013;14(2):126-35

7. Geers AL, Wellman JA, Helfer SG, Fowler SL, France CR. Dispositional optimism and thoughts of wellbeing determine sensitivity to an experimental pain task. . Annals of Behavioral Medicine. 2008(36):304-13.

8. Carver CS, Scheier MF, Segerstrom SC. Optimism. Clinical psychology review. 2010;30(7):879-89.

9. Costello NL, Bragdon EE, Light KC, Sirgudsson A, Bunting S, Grewen K, et al. Temporomandibular disorder and optimism: relationships to ischemic pain sensitivity and interleukin-6. Pain. 2002(100):99-110.

10. Allison PJ, Guichard C, Gilain L. A prospective investigation of dispositional optimism as a predictor of health-related quality of life in head and neck cancer patients. Qual Life Res. 2000;9(8):951-60.

11. Achat H, Kawachi I, Spiro A, zrd, DeMolles DA, Sparrow D. Optimism and depression as predictors of physical and mental health functioning: the Normative Aging Study. Annals of behavioral medicine : a publication of the Society of Behavioral Medicine. 2000;22(2):127-30.

12. Sipila K, Ylostalo PV, Ek E, Zitting P, Knuuttila ML. Association between optimism and self-reported facial pain. Acta Odontol Scand. 2006;64(3):177-82.

13. Staub E, Tursky B, Schwartz GE. Self-control and predictability: their effects on reactions to aversive stimulation. J Pers Soc Psychol. 1971;18(2):157-62.

14. Price DD. Psychological and neural mechanisms of the affective dimension of pain. Science. 2000;288(5472):1769-72.

15. Dannecker EA, Price DD, Robinson ME. An examination of the relationships among recalled, expected, and actual intensity and unpleasantness of delayed onset muscle pain. J Pain. 2003;4(2):74-81.

16. Robinson ME, Gagnon CM, Riley JL, 3rd, Price DD. Altering gender role expectations: effects on pain tolerance, pain threshold, and pain ratings. J Pain. 2003;4(5):284-8.

17. Medoc. TSA II; NeuroSensory Analyzern.d.

18. Angst MS, Tingle M, Phillips NG, Carvalho B. Determining heat and mechanical pain threshold in inflamed skin of human subjects. J Vis Exp. 2009(23). 
19. Scheier MF, Carver CS, Bridges MW. Distinguishing optimism from neuroticism (and trait anxiety, selfmastery, and self-esteem): a reevaluation of the Life Orientation Test. J Pers Soc Psychol. 1994;67(6):1063-78

20. Baron RM, Kenny DA. The moderator-mediator variable distinction in social psychological research: Conceptual, strategic, and statistical considerations. Journal of Personality and Social Psychology. $1986 ; 51(6): 1173-82$.

21. Ronaldson A, Poole L, Kidd T, Leigh E, Jahangiri M, Steptoe A. Optimism measured pre-operatively is associated with reduced pain intensity and physical symptom reporting after coronary artery bypass graft surgery. J Psychosom Res. 2014;77(4):278-82.

22. Scheier MF, Carver CS. Optimism, coping, and health: assessment and implications of generalized outcome expectancies. Health Psychol. 1985;4(3):219-47. 